\title{
A high resolution scheme for flows in open channels with arbitrary cross-section
}

\author{
Elisa Aldrighetti ${ }^{1}$ and Paola Zanolli ${ }^{1}$ \\ Department of Mathematics - University of Trento, \\ Via Sommarive 14, I-38050 Povo (Trento), Italy \\ E-mail: aldrighe@science.unitn.it, zanolli@science.unitn.it
}

\begin{abstract}
SUMMARY
The aim of this paper is to present a numerical scheme to simulate unsteady, one dimensional flows in open channels with arbitrary cross-section. This scheme is fully conservative of volume and momentum and preserves the non-negativity of the water depth. The finite difference method derived is semiimplicit in time and based on a space staggered grid. A high resolution technique, the flux limiter method, is implemented to control the accuracy of the proposed scheme. Our purpose is to achieve the precision and the stability of the method with respect to the regularity of the data. A few computational examples on classical test cases are given to illustrate the properties of the present method in terms of stability, accuracy and efficiency.
\end{abstract}

KEY WORDS: shallow water; conservation properties; finite difference; semi-implicit; staggered grids

\section{INTRODUCTION}

Unsteady, one dimensional flows in open channels or rivers are governed by the one dimensional Saint Venant equations, a particular case of the shallow water equations. These equations are

\footnotetext{
${ }^{1}$ Correspondence to: Department of Mathematics - University of Trento, Via Sommarive 14, I-38050 Povo (Trento), Italy.
} 
derived from the physical principles of conservation of mass and momentum (see, e.g., [1][5]).

Assuming an arbitrary cross section, these equations describe the flow through the crosssectional averaged water velocity $u(x, t)$ and the instantaneous water surface elevation $\eta(x, t)$ measured vertically from a reference datum. These two unknowns are related by the following hyperbolic system of two partial differential equations

$$
\begin{gathered}
A_{t}+q_{x}=0 \\
q_{t}+(u q)_{x}+g A \eta_{x}+\gamma u=0,
\end{gathered}
$$

where $A(x, \eta)$ is an arbitrary but prescribed function of space and water surface elevation representing the cross-section area; $\gamma$ is a non-negative friction coefficient; $g$ is the gravity acceleration; $q=A u$ is the momentum.

In the current literature several numerical techniques for solving equations (1)-(2) are known. These include the method of characteristics, explicit difference methods, fully implicit methods, Godunov methods [7] and semi-implicit methods [2]. In particular, the method of characteristics is very efficient in the treatment of boundary conditions, but does not guarantee volume and momentum conservation. The Godunov's type methods (see, e.g., [12]) instead, require the solution of local Riemann problems and, consequently, are very effective on simple channel geometries with flat, horizontal bottom and rectangular cross section. For space varying bottom profiles, however, the bottom slope appears as a source term that may generate artificial flows [12] unless specific treatments of the geometrical source terms are implemented [6]. Moreover, Godunov's type methods are explicit in time and, accordingly, the allowed time step is restricted by a C.F.L. stability condition. Alternatively, semi-implicit methods (see, e.g., [3]) can be unconditionally stable and computationally efficient. These methods, however, when they do not satisfy momentum conservation, may produce incorrect results if applied to extreme problems having a discontinuous solution. The semi-implicit method recently presented by Stelling in [11] combines the efficiency of staggered grids with conservation properties and can be applied to problems including rapidly varying flows. This method, however, is suitable only for rectangular channels. Its formulation is quite complicated and has to switch between momentum and energy head conservation depending on local flow 
condition (see reference [11] for details). A semi-implicit method that conserves the fluid volume when applied to channels with arbitrary cross sections was presented in [5].

In the present paper a numerical technique to solve equations (1)-(2) is derived, discussed and applied. This technique is fully conservative, satisfies a correct momentum balance near large gradients and, under a suitable constraint on the time interval, ensures the non-negativity of the water volume, so allowing a correct solution of problems presenting flooding and drying. A proper semi-implicit discretization leads to a scheme that is relatively simple and highly accurate, even if the C.F.L. condition is violated.

This paper is organized in 8 sections. From the second to the sixth the proposed numerical technique is described. In Section 7, a few computational examples on the classical dam break and hydraulic jump tests are given to illustrate some properties of the present method.

\section{SEMI-IMPLICIT FORM AND NOTATION}

In order to obtain an efficient numerical method that does not "suffer" from stability problems, some terms in the governing equations are discretized implicitly. The determination of the specific form of the semi-implicit discretization follows directly from the analysis of the hyperbolic system (1)-(2) and from the study of the C.F.L. stability condition [2].

Hence, in the momentum equation the free surface slope is discretized with the $\theta$ method and the friction term is taken implicitly [3]. In the continuity equation the $\theta$ method is chosen for the spatial derivative. The remaining terms are discretized explicitly [4][10]. For stability reasons [3], $\theta$ is considered in $\left[\frac{1}{2}, 1\right]$.

Equations (1) and (2) are discretized in the spatial interval $[0, L]$ on a space staggered grid whose nodes are denoted by $x_{i}$ and $x_{i+1 / 2}$. The discrete velocity $u_{i+1 / 2}$ (or the momentum $\left.q_{i+1 / 2}\right)$ is defined at half integer nodes and the discrete surface elevation $\eta_{i}$, assumed to be constant in the interval $\left[x_{i-1 / 2}, x_{i+1 / 2}\right]$, is defined at integer nodes. The grid intervals are denoted by $\Delta x_{i}=x_{i+1 / 2}-x_{i-1 / 2}$ and $\Delta x_{i+1 / 2}=\frac{\Delta x_{i+1}+\Delta x_{i}}{2}$. The time interval is taken to be $\Delta t$. 


\section{A CONSERVATIVE SCHEME FOR THE CONTINUITY EQUATION}

The continuity Equation (1) expresses the physical law of conservation of volume and it is discretized by a finite volume method in space and by the $\theta$ method in time [5]. Specifically, from the integration in space of (1) over the interval $\left[x_{i-1 / 2}, x_{i+1 / 2}\right]$ and from the discretization in time, it follows

$$
V_{i}\left(\eta_{i}^{n+1}\right)=V_{i}\left(\eta_{i}^{n}\right)-\Delta t\left[q_{i+1 / 2}^{n+\theta}-q_{i-1 / 2}^{n+\theta}\right]
$$

where the fluid volume $V_{i}\left(\eta_{i}\right)=\int_{x_{i-1 / 2}}^{x_{i+1 / 2}} A d x$ is, in general, a non linear function of $\eta$ and $q^{n+\theta}=\theta q^{n+1}+(1-\theta) q^{n}$.

Equation (3) obviously expresses a discrete conservation of fluid volume.

The particular attention given here to volume conservation is justified by the importance of this conservation when the channel has a non-rectangular cross-section. In this case, traditional numerical methods (and even the Godunov's type methods) apply a linearization technique to the non linear function $V$ in equation (3). Specifically,

$$
V_{i}\left(\eta_{i}^{n+1}\right) \approx V_{i}\left(\eta_{i}^{n}\right)+\frac{\partial V_{i}\left(\eta_{i}^{n}\right)}{\partial \eta}\left(\eta_{i}^{n+1}-\eta_{i}^{n}\right)
$$

where $\frac{\partial V_{i}\left(\eta_{i}^{n}\right)}{\partial \eta}$ represents the surface area between $x_{i-1 / 2}$ and $x_{i+1 / 2}$.

Substitution of (4) into (3) yields

$$
\frac{\partial V_{i}\left(\eta_{i}^{n}\right)}{\partial \eta}\left(\eta_{i}^{n+1}-\eta_{i}^{n}\right)+\Delta t\left[q_{i+1 / 2}^{n+\theta}-q_{i-1 / 2}^{n+\theta}\right]=0,
$$

where the term $\frac{\partial V_{i}\left(\eta_{i}^{n}\right)}{\partial \eta}\left(\eta_{i}^{n+1}-\eta_{i}^{n}\right)$ is no longer the volume variation unless $\frac{\partial V_{i}}{\partial \eta}$ is a constant. This is the case, e.g. for channels with rectangular cross section. In general, however, the linearized equation (5) or similar linearizations, will not guarantee volume conservation and an artificial loss or creation of mass may result.

From equation (3), one can derive a condition for the non-negativity of the water volume, i.e.

$$
\left[q_{i+1 / 2}^{n+\theta}-q_{i-1 / 2}^{n+\theta}\right] \Delta t \leq V_{i}\left(\eta_{i}^{n}\right) .
$$




\section{A CONSERVATIVE SCHEME FOR THE MOMENTUM EQUATION}

Equation (2) is discretized with a conservative method in order to obtain a physically correct solution also under extreme circumstances. Specifically, centered finite differences are used for the integration in space of water surface elevation, while the semi-implicit method is used for the time integration (see, e.g. References [2][3][4][5]):

$$
\left(1+\gamma_{i+1 / 2}^{n} \Delta t\right) q_{i+1 / 2}^{n+1}+g A_{i+1 / 2}^{n} \theta \Delta t \frac{\left(\eta_{i+1}^{n+1}-\eta_{i}^{n+1}\right)}{\Delta x_{i+1 / 2}}=F_{i+1 / 2}^{n}
$$

where

$$
F_{i+1 / 2}^{n}=q_{i+1 / 2}^{n}-\Delta t \frac{\left[(u q)_{i+1}^{n}-(u q)_{i}^{n}\right]}{\Delta x_{i+1 / 2}}-g A_{i+1 / 2}^{n}(1-\theta) \Delta t \frac{\left(\eta_{i+1}^{n}-\eta_{i}^{n}\right)}{\Delta x_{i+1 / 2}}
$$

is a finite difference operator including the explicit discretizations of the advective and the free surface slope terms; $A_{i+1 / 2}^{n}=A\left(x_{i+1 / 2}, \frac{\eta_{i+1}^{n}+\eta_{i}^{n}}{2}\right)$.

Here, it is worth noting that in case of a frictionless channel with rectangular cross section and flat bottom one has $A(x, \eta)=B H=B(h+\eta)$, where $B$ is the channel width and $h=$ constant is the channel depth when $\eta=0$. In this case, Equation (7) can be regarded as being the semi-implicit time discretization of

$$
\frac{d q_{i+1 / 2}}{d t}+\frac{(u q)_{i+1}-(u q)_{i}}{\Delta x_{i+1 / 2}}=-g B \frac{\left(H_{i+1}+H_{i}\right)}{2} \frac{\left(H_{i+1}-H_{i}\right)}{\Delta x_{i+1 / 2}}
$$

or equivalently,

$$
\frac{d q_{i+1 / 2}}{d t}+\frac{(u q)_{i+1}-(u q)_{i}}{\Delta x_{i+1 / 2}}=-\frac{g B}{2} \frac{\left(H_{i+1}^{2}-H_{i}^{2}\right)}{\Delta x_{i+1 / 2}} .
$$

Interestingly enough, even though the given momentum Equation (2) is not written in conservative form, the resulting Equation (10) represents the precise momentum conservation because it is written in flux form (see, e.g. Reference [11] for further details).

We shall then assume that the more general equation (7) is conservative also in the more general case of channels with arbitrary cross section and with varying bottom slope. 


\section{THE SOLUTION ALGORITHM}

At each time step Equations (3) and (7) form a system of non linear equations with unknowns $q_{i+1 / 2}^{n+1}$ and $\eta_{i}^{n+1}$ over the entire computational mesh. This system can be reduced for computational convenience to a smaller one in which $\eta_{i}^{n+1}$ are the only unknowns. Specifically, the expression for $q_{i \pm 1 / 2}^{n+1}$ can be substituted from (7) into (3) to obtain

$$
V_{i}\left(\eta_{i}^{n+1}\right)+p_{i-1 / 2}^{n} \eta_{i-1}^{n+1}+d_{i}^{n} \eta_{i}^{n+1}+p_{i+1 / 2}^{n} \eta_{i+1}^{n+1}=f_{i}^{n}
$$

where $p_{i \pm 1 / 2}^{n}, d_{i}^{n}$ and $f_{i}^{n}$ are

$$
\begin{gathered}
p_{i \pm 1 / 2}^{n}=-\frac{g(\theta \Delta t)^{2} A_{i \pm 1 / 2}^{n}}{\Delta x_{i+1 / 2}\left(1+\gamma_{i+1 / 2}^{n} \Delta t\right)} \quad d_{i}^{n}=-p_{i+1 / 2}^{n}-p_{i-1 / 2}^{n}, \\
f_{i}^{n}=V_{i}\left(\eta_{i}^{n}\right)-(1-\theta) \Delta t\left[q_{i+1 / 2}^{n}-q_{i-1 / 2}^{n}\right]-\theta \Delta t\left[\frac{F_{i+1 / 2}^{n}}{\left(1+\gamma_{i+1 / 2}^{n} \Delta t\right)}-\frac{F_{i-1 / 2}^{n}}{\left(1+\gamma_{i-1 / 2}^{n} \Delta t\right)}\right]
\end{gathered}
$$

For every time step $n$, system (11) can be written in a more compact matrix notation, as follows

$$
\mathbf{V}(\eta)+\mathbf{M} \eta=\mathbf{f},
$$

where $\eta=\left(\eta_{1}, \eta_{2}, \ldots, \eta_{N}\right)^{T}$ is the vector of the unknowns,

$$
\mathbf{V}(\eta)=\left(\begin{array}{c}
V_{1}\left(\eta_{1}\right) \\
V_{2}\left(\eta_{2}\right) \\
\ldots \\
V_{N}\left(\eta_{N}\right)
\end{array}\right), \quad \mathbf{M}=\left(\begin{array}{cccc}
d_{1} & p_{\frac{3}{2}} & \ldots & 0 \\
p_{\frac{3}{2}} & \ddots & \ddots & \vdots \\
\vdots & \ddots & \ddots & p_{N-\frac{1}{2}} \\
0 & \ldots & p_{N-\frac{1}{2}} & d_{N}
\end{array}\right), \quad \mathbf{f}=\left(\begin{array}{c}
f_{1} \\
f_{2} \\
\ldots \\
f_{N}
\end{array}\right) .
$$

System (11) is mildly non linear. The coefficient matrix $\mathbf{M}$ is symmetric, tridiagonal, diagonally dominant with positive elements on the main diagonal and negative ones elsewhere.

Regarding the nonlinear part, we assume that $\mathbf{V}$ is Lipschitz continuous, that is $V_{i}$ is Lipschitz continuous with constant $L_{i}$ for each $i=1, \ldots N$ so that $\mathbf{L}=\operatorname{diag}\left(L_{1}, L_{2}, \ldots L_{N}\right)$ is the Lipschitz constant of $\mathbf{V}$.

This is a realistic assumption, because it means that the surface area $\frac{\partial V_{i}}{\partial \eta}$ is always bounded for every $\eta$ and thus the flow is always assumed to be confined within the channel banks. 
It is known that under this hypothesis, the existence and the uniqueness of the solution of system (12) is assured [9] and can be computed by using, for example, the iterative method presented in Reference [5].

Once the solution for $\eta^{n+1}$ has been determined, $q^{n+1}$ can be easily computed by substitution $\eta^{n+1}$ in (7).

\section{ADVECTIVE TERMS}

The value of $u q$ at the integer node $i$, as required by $F$, may be chosen with an upwind rule as follows:

$$
(u q)_{i}=\left\{\begin{array}{lll}
u_{i-1 / 2} q_{i-1 / 2} & \text { if } & \frac{u_{i-1 / 2}+u_{i+1 / 2}}{2} \geq 0 \\
u_{i+1 / 2} q_{i+1 / 2} & \text { if } & \frac{u_{i-1 / 2}+u_{i+1 / 2}}{2}<0 .
\end{array}\right.
$$

With this choice the resulting numerical scheme is only first order accurate. In general, all first order schemes suffer from numerical dissipation and all second order schemes suffer from artificial dispersion, which creates oscillations around discontinuities.

In order to improve the accuracy without running into stability problems but leading it to satisfy the TVD property [12], the flux limiter method has been used. This high order resolution method switches between a second order approximation when the data are sufficiently smooth and a first order approximation near a discontinuity.

The flux limiter method has been implemented in the approximation of the advective term in the momentum Equation (7). Starting from the proposed discretization, the flux limiter approach adds to the numerical flux $(u q)_{i}$ a correction term limited by a flux limiter function $\Psi$ that depends on the regularity of the data. Thus, the approximation of the advective term can be rewritten as

$$
(u q)_{x} \approx \frac{\left[(u q)_{i+1}+\frac{1}{2} \Psi\left(r_{i+\frac{3}{2}}\right) \Delta(u q)_{i+3 / 2}\right]-\left[(u q)_{i}+\frac{1}{2} \Psi\left(r_{i+\frac{1}{2}}\right) \Delta(u q)_{i+1 / 2}\right]}{\Delta x_{i+1 / 2}}
$$

where $(u q)_{i}$ is given by (14), the corrective term $\Delta(u q)_{i+1 / 2}$ is taken to be

$$
\Delta(u q)_{i+1 / 2}=\left\{\begin{array}{llll}
(u q)_{i+1 / 2}-(u q)_{i-1 / 2} & \text { if } & \frac{u_{i+1 / 2}+u_{i-1 / 2}}{2} \geq 0 \\
(u q)_{i+3 / 2}-(u q)_{i+1 / 2} & \text { if } & \frac{u_{i+1 / 2}+u_{i-1 / 2}}{2}<0
\end{array}\right.
$$


and the smoothness of the data in $x_{i+1 / 2}$ is defined as

$$
r_{i+1 / 2}=\left\{\begin{array}{lll}
\frac{u_{i-1 / 2}-u_{i-3 / 2}}{u_{i+1 / 2}-u_{i-1 / 2}} & \text { if } & \frac{u_{i+1 / 2}+u_{i-1 / 2}}{2} \geq 0 \\
\frac{u_{i+1 / 2}-u_{i-1 / 2}}{u_{i+3 / 2}-u_{i+1 / 2}} & \text { if } & \frac{u_{i+1 / 2}+u_{i+1 / 2}}{2}<0 .
\end{array}\right.
$$

The flux limiting function $\Psi$ can be chosen in several ways (see, e.g., [8] for details).

\section{NUMERICAL RESULTS}

The first test problem is the well known dam break problem in a $1 \mathrm{~m}$ long rectangular channel over a frictionless dry bed. The initial conditions are

$$
u(x, 0)=0, \quad \eta(x, 0)=\left\{\begin{array}{lll}
\eta_{l} & \text { if } & 0 \leq x \leq \frac{1}{2} \\
\eta_{r} & \text { if } & \frac{1}{2}<x \leq 1 .
\end{array}\right.
$$

The physical and computational parameters are $\eta_{l}=1 \mathrm{~m}, \eta_{r}=0 \mathrm{~m}, \gamma=0, g=1 \mathrm{~m} / \mathrm{s}^{2}$, $\Delta x=0.005 m, \theta=1$ and $\Delta t=10^{-3} s$. In this example $\eta(x, t)$ also represents the total water depth which is initially zero for $\frac{1}{2} \leq x \leq 1$.

Figure $(a)$ shows the numerical results and the analytical solution (plotted with a dotted line) at time $T=0.15 \mathrm{~s}$. These results compare favorably well with those obtained from highorder Godunov's type methods (see, e.g., [12]).

The second test problem is a dam break problem in a channel with flat bottom and triangular section of area $A=10 \eta^{2}$. The initial conditions are the same as in (18) with $\eta_{l}=1 \mathrm{~m}$ and $\eta_{r}=0.1 \mathrm{~m}$. The computational parameters are set as in the first example except for $\theta=0.5$.

The results obtained at time $T=0.3 s$ are plotted in Figure $(b)$. This example shows the applicability of the present algorithm to a dam break problem where precise volume conservation is essential and not easily obtained by traditional linear schemes.

The last example is a hydraulic jump test problem in a $100 \mathrm{~m}$ long rectangular channel. In the middle of the channel there is a sill with a crest of $1 \mathrm{~m}$ height and $10 \mathrm{~m}$ long and the tangent of slopes at both sides is 0,2 . There are two open boundaries, the inflow and the outflow, where a discharge of $1 \mathrm{~m}^{3} / \mathrm{s}$ and a water depth of $1 \mathrm{~m}$, respectively, are imposed [11]. 
The discretization parameters are $\gamma=0, g=9.81 \mathrm{~m} / \mathrm{s}^{2}, \Delta x=0.5 \mathrm{~m}, \theta=1$ and $\Delta t=10^{-3} \mathrm{~s}$. This is a severe test case that involves a problem with a varying bottom profile.

The steady state of the free surface of this phenomenon is plotted in Figure $(c)$. These results also compare favorably well with those obtained by Stelling and Duinmeijer [11].

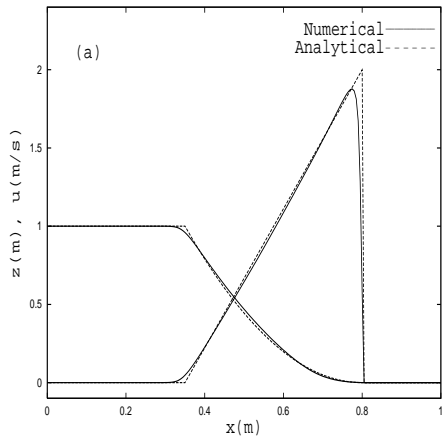

(a) Dam break over a dry bed.

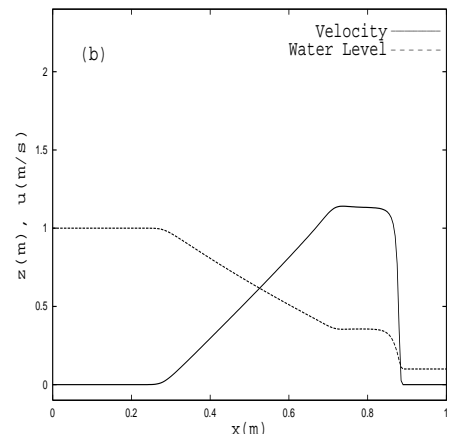

(b) Dam break over a wet bed.

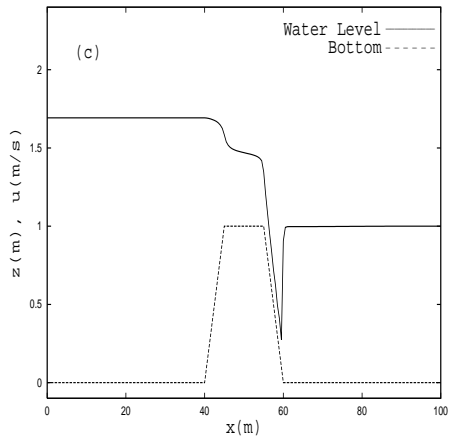

(c) Hydraulic jump.

Comparisons with first order methods show that the present results are definitely sharper.

\section{CONCLUSIONS}

A fully conservative semi-implicit finite difference method to solve the Saint Venant equations has been presented, discussed and applied. It is simple, volume and momentum conservative and preserves the non negativity of the water volume. A flux limiter technique has been implemented to deal with steep gradients like the ones that are found in dam break problems. Some numerical results are discussed. The extension of this method to two spatial dimensions is left for a future work.

\section{References}

[1] Abbott MB. Computational Hydraulics. Worcester: Ashgate, 1992.

[2] Casulli V. Semi-Implicit Finite Difference Methods for the Two-Dimensional Shallow Water Equations. Journal of Computational Physics. 1990; 86:56-74. 
[3] Casulli V, Cattani E. Stability, accuracy and efficiency of a semi-implicit method for three dimensional shallow water flows. Computers $E_{3}$ Mathematics with Application. 1994; 15(6):629-648.

[4] Casulli V, Cheng RT. Stability Analysis of Eulerian-Lagrangian Method for the OneDimensional Shallow-Water Equations. Applied Mathematical Modelling. 1990; 14:122131.

[5] Casulli V, Zanolli P. A conservative Semi-Implicit Scheme for Open Channel Flows. International Journal of Applied Science \& Computations. 1998; 5: 1-10.

[6] Garcia-Navarro P, Vazquez-Cendon ME. On numerical treatment of the source terms in the shallow water equations. Computers \& Fluids. 2000; 29: 951-979.

[7] Hirsh C. Numerical Computation of Internal and External Flows. Volume 2: Computational Methods for Inviscid and Viscous Flows. John Wiley \& Sons Ltd, 1990.

[8] LeVeque RJ. Finite Volume Methods for hyperbolic Problems. Cambridge University Press, 2002.

[9] Ortega JM, Rheimboldt WC. Iterative Solution of Non-Linear Equation in several variables. Academics Press: New York, 1970.

[10] Roache PJ. Computational Fluid Dynamics. Hermosa Pub.: Albuquerque, 1972.

[11] Stelling GS, Duinmeijer SPA. A staggered conservative scheme for every Froude number in rapidly varied shallow water flows. International Journal for Numerical Methods in Fluids. 2003; 43:1329-1354.

[12] Toro EF. Shock capturing Methods for free surface shallow flows. John Wiley \& Sons Ltd, 2001. 